\title{
Temperature Measurement by Statistical Parameters of Ultrasound Signal Backscattered from Tissue Samples
}

\author{
B. Gambin* and E. Kruglenko \\ Institute of Fundamental Technological Research Polish Academy of Sciences, \\ Pawińskiego 5B, 02-106 Warszawa, Poland
}

\begin{abstract}
A novel estimation of temperature changes inside soft tissues has been proposed in sub-ablation range, i.e. $\approx 20^{\circ} \mathrm{C}-48^{\circ} \mathrm{C}$. This estimation has been obtained by studying statistical properties of backscattered ultrasonic signals. Two different procedures of heating/cooling have been performed in which the RF echoes have been registered from soft tissue phantom in the first procedure, and from soft tissue in vitro in the second one. Calculated envelopes of signals registered in time points during heating/cooling experiments have been treated as a statistical sample drawn from a random variable with three different distributions, namely the Rayleigh distribution, the Nakagami distribution, and the $K$-distribution. The histograms obtained in subsequent time moments have been fitted to the three distributions. Dependencies of their shape and scale parameters on temperature have been calculated. It is concluded that the shape parameter of the $K$-distribution can be chosen as the best marker of temperature changes in both experiments. The choice of the marker has been made by analysis of temperature dependencies of all calculated parameters and by comparing the quality of fitting all histograms to the considered distributions. Besides, the chosen marker as a function of temperature exhibits the closest shape to temperature/time function experimentally measured.
\end{abstract}

DOI: 10.12693/APhysPolA.128.A-72

PACS: 87.50.wp, 43.35. + d, 87.57.-S

\section{Introduction}

To date, there are no non-invasive clinically used medical procedures for measuring temperature rise inside soft tissues. Ultrasonic imaging techniques are predisposed to be used in measurements of temperature inside living soft tissues because ultrasounds do not cause ionization in living cells, so in that sense they can be considered safe, cf. [1]. Different acoustic measurement methods of temperature changes have been studied in the last decades, e.g. [1-3]. Most of these studies concern the range of temperatures much above $43{ }^{\circ} \mathrm{C}$ and they are associated with the control of the course of High Intensity Focused Ultrasound (HIFU) treatment procedures.

In this post ablation range of temperature, the changes in soft tissue cells are strong, denaturation of proteins occurs, and the heated region can be visible on USG images.

In the paper we are interested in the lower temperature range; in our experiments this is the range from $20^{\circ} \mathrm{C}$ to $48^{\circ} \mathrm{C}$. Heating the tissues up to $43^{\circ} \mathrm{C}$ guarantees that all living functions of cells are preserved and moreover, the small amount of thermal energy can have a positive effect on many biochemical processes such as e.g. a gene expression or increasing level of HSPs (heat shock proteins). The HSPs, among other functionalities, are used by living cells in processes of repairing damaged proteins in neurodegenerative diseases. The heating procedure of target regions inside tissues by using LIFU (law intensity focused ultrasound) are studied in [4-6]. In the latter

*corresponding author; e-mail: bgambin@ippt.gov.pl of the quoted papers, the increase of HSP level is correlated with temperature changes. Additionally, the lowlevel hyperthermia can be an adjuvant therapy for drug delivery applications, as well as for more traditional applications such as radio sensitization [7-8]. The authors of the second paper discussed also the manner in which focused ultrasound exposures at low rates of energy deposition are being developed for a variety of clinically translatable applications for the treatment of cancer.

It is a well-documented fact that the sub-ablation temperature range causes changes in the acoustic wave velocity. The ultrasonic measurement of temperature is mostly based on studying the impact of changes in the sound velocity on properties of the backscattered signal (see the updated review paper [9] devoted to the thermal strain imaging).

On the other hand, the mechanical nature of ultrasound results in existence of strong correlation between backscattered signal and tissues microstructural properties. This correlations are extensively used in modern methods of ultrasound tissue differentiation (see updated discussion of QUS (quantitative ultrasound) in [1]). The physical principle which is the main motivation of our study is the fact that besides already exploited changes of the sound speed, temperature increase/decrease must follow the reorganization spatial distribution of scatterers in the tissue on different size levels and, independently, it may cause the changes in physical properties of scatterers, particularly their reflectivity. Therefore, temperature-induced microstructural changes should be "visible" in the backscattered signals statistics. The papers with primary results important for the study and close to this idea have been published by 
us in $[4,10-15]$. Apart from our own papers, we have found only one paper in which the statistical properties of RF signal envelope have been also studied to describe temperature changes [16]. The authors did not discuss the choice of the Nakagami distribution function as a randomness descriptor of echo envelopes data collected in the special heating experiment.

It is worth underlining that B-scan images of our experiments shown in Fig. 1 do not carry any information of visible structural changes in soft tissues during thermal process for the sub-ablation temperature range studied here. We have decided to search changes in statistics of the backscattered signal. We would like to predict, as precisely as possible, the way in which the temperature changes inside soft tissue phantom and soft tissue samples in vitro. The link between temperature level and some selected statistical parameters of the signal envelope is discussed. Our analysis is limited to finding the ultrasonic characteristics of temperature changes within the heated regions of two types of samples and two different heating procedures. At first, we perform heating and cooling process using the soft tissue mimicking material made from Polyvinyl Alcohol cryogel (PVA-c). Some of its acoustic properties are similar to those of the soft tissue because PVA-c has a specific kind of microstructure with multilevel organization. The main difference is a very small absorption of acoustic energy, so this kind of material cannot be heated by the ultrasound beam. Nevertheless, the thermal behavior of PVA-c is stable enough up to about $50^{\circ} \mathrm{C}$, so we have decided to heat the sample in a water bath. In the second experiment, soft tissue samples in vitro have been heated by an ultrasonic beam with the transducer supplied with two different powers. In parallel, during the heating, the USG scanning of the sample have been performed with the help of other standard imaging transducer and the data were collected with the Synthetic Transmit Aperture (STA) method [17].

The experiments are described in Sect. 2. The statistical analysis of RF signals has been performed in Sect. 3. First, filtering of signals out from the band of transmitted frequencies have been performed. Then, the analytical signals and the envelope histograms have been calculated. The different probability distributions, namely the Rayleigh distribution, the Nakagami distribution, and the $K$-distribution have been compared to the family of histograms parameterized by time in two well-defined periods of experiments performed. Five parameters, one for the Rayleigh distribution and two for each of the other two distributions as functions of time have also been obtained.

The choice of the best statistical parameter evaluating temperature changes will be based on two criteria: the histograms matching and the strongest sensitivity of their parameter to temperature changes at two thermal processes in two materials samples.

The time scale has been converted to temperature scales consistently to the experimentally measured quantities. Taking into account dependencies on temperature of different parameters, we conclude in Sect. 4 that the optimal scalar measure of temperature level from among the five studied quantities is the shape parameter of the $K$-distribution.
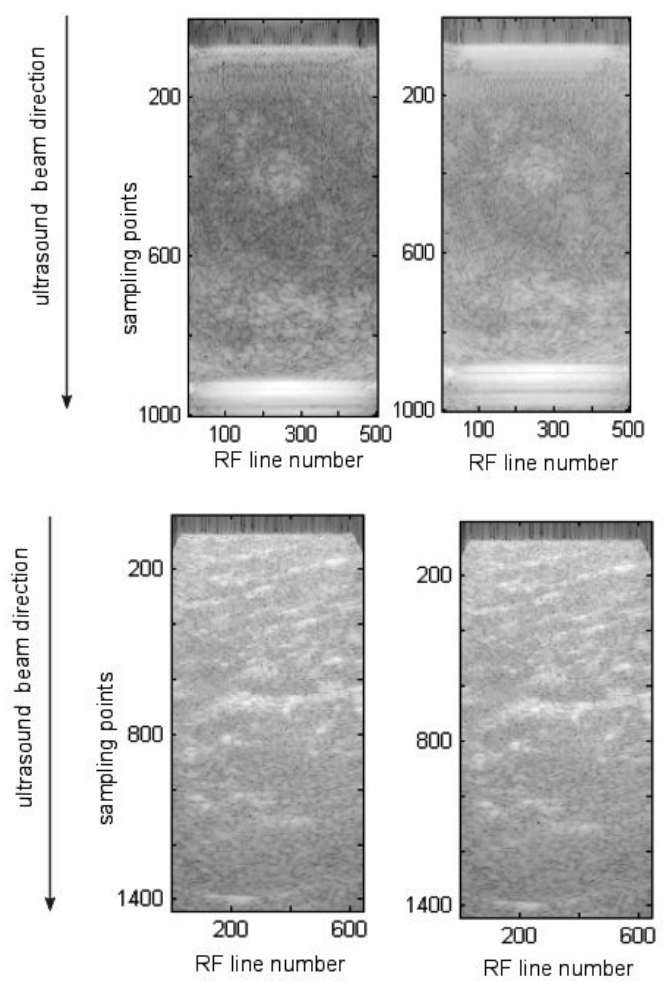

Fig. 1. (a) B-mode image of PVA-c phantom on different temperature levels. Left - at the beginning of experiment, temperature $-20.6^{\circ} \mathrm{C}$; right - after $1 \mathrm{~h}$ of heating, temperature $-48.8^{\circ} \mathrm{C}$; (b) B-mode image of soft tissue sample on different temperature levels. Left - at the beginning of experiment, temperature $20.6{ }^{\circ} \mathrm{C}$; right - after 10 min of heating with power $6 \mathrm{~W}$, temperature $-47^{\circ} \mathrm{C}$.

\section{Experiments}

The sample made from PVA-c, $40 \mathrm{~mm} \times 40 \mathrm{~mm} \times 8 \mathrm{~mm}$, has been immersed in a water bath and subjected to uniform heating. The thermostat was set so that within one hour the water temperature registered by the thermometer increased linearly from $20.6^{\circ} \mathrm{C}$ to $48.8^{\circ} \mathrm{C}$, see Fig. 1 . Next, the thermostat was disabled and the temperature of the water decreased to $45.8^{\circ} \mathrm{C}$ after two hours. Note that the whole volume of the sample has been heated uniformly. The temperature distribution during heating/cooling process within the sample was calculated using FEM program implemented as part of the Abaqus 6.12 software (DS Simulia Corp.) [4, 10], see Fig. 2.

Backscattered ultrasound signals have been collected with the use of transducer L14-5/38 ULTRASONIX at the frequency of $8 \mathrm{MHz}$ (ULTRASONIX SonixTOUCH, British Columbia, Canada). The transmitted pulse comprised 2 periods of the sine wave (pulse duration of 

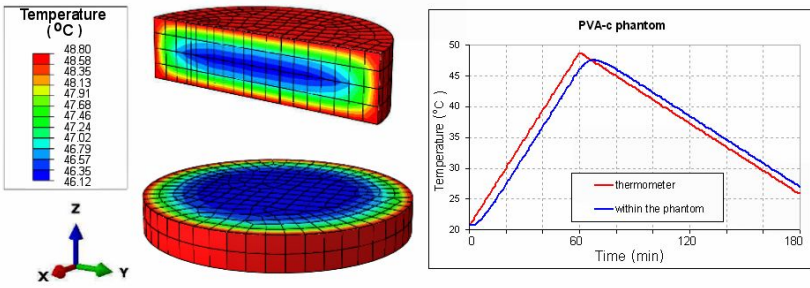

Fig. 2. Left: Calculated temperature after $1 \mathrm{~h}$ of heating in different points of the PVA-c sample. Right: The measured (red line) and calculated (blue line) temperature as a function of time during the PVA-c heating/cooling process.

0.25 microseconds). To collect the data, the Synthetic Transmit Aperture (STA) method has been used [14, 15]. With the method, an ultrasonic signal was transmitted by a single transducer and received echoes were registered by all elements of the transducer array. Focusing took place at every point B-mode data.

The system for heating the soft tissue samples in vitro consisted of a generator (Agilent 332, Aprings Colorado, USA), an amplifier (ENI 1325LA, Rochester NY, USA), a spherical ultrasonic transducer (central frequency $2.2 \mathrm{MHz}$, diameter $44 \mathrm{~mm}, 44.5 \mathrm{~mm}$ focal length, area $S=15.2 \mathrm{~cm}^{2}$ ), and an oscilloscope (Tektronix TDS3012B), see Fig. 3.
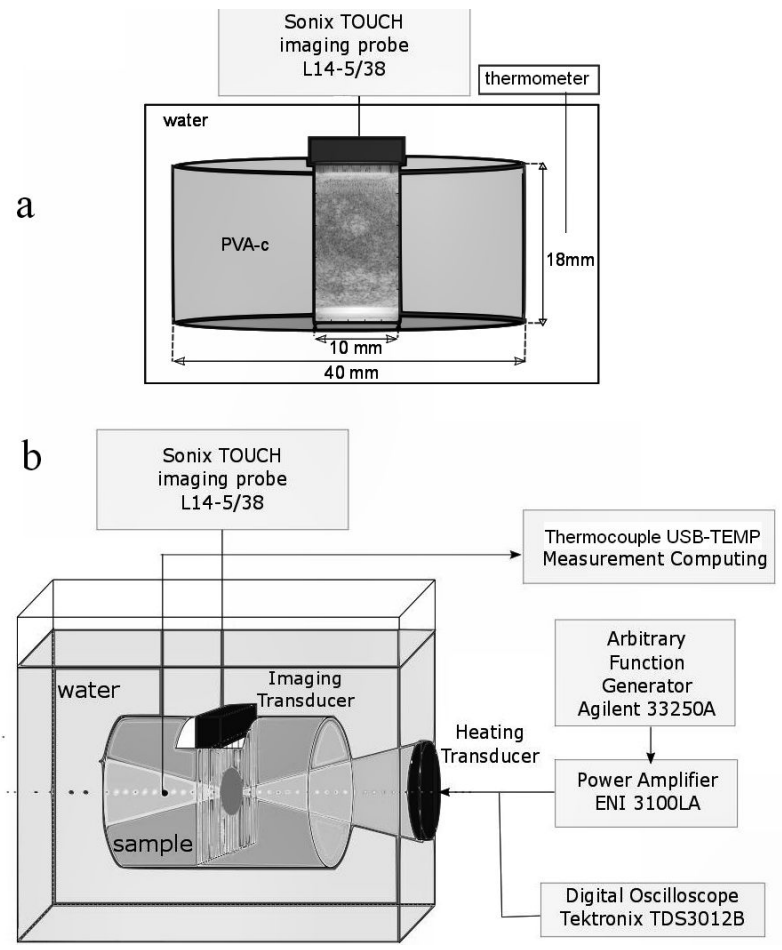

Fig. 3. Scheme of experiments: (a) for PVA-c; (b) for soft tissue samples.

Irradiation with two different powers, $4 \mathrm{~W}$ and $6 \mathrm{~W}$, has been performed. During 10 minutes of heating and 10 minutes of cooling, the temperature changes were recorded using thermocouples and registered by the module (USB-TEMP, Measurement Computing, Norton, USA).

The temperature within the sample has been measured along the beam axis at different distances from the head. The geometrical focus was located about $25 \mathrm{~mm}$ from the surface of the transducer, while the maximum temperature observed in the pattern was at a distance of $25 \mathrm{~mm}$, i.e. virtually at the same point. The linear transducer (L14-5/38) located across the heating beam at a distance of $25 \mathrm{~mm}$ from the transmitter has been used to produce images during heating by the focused transducer. In this case, the heated volume was concentrated near the focusing area inside the sample and the temperature distribution was inhomogeneous.

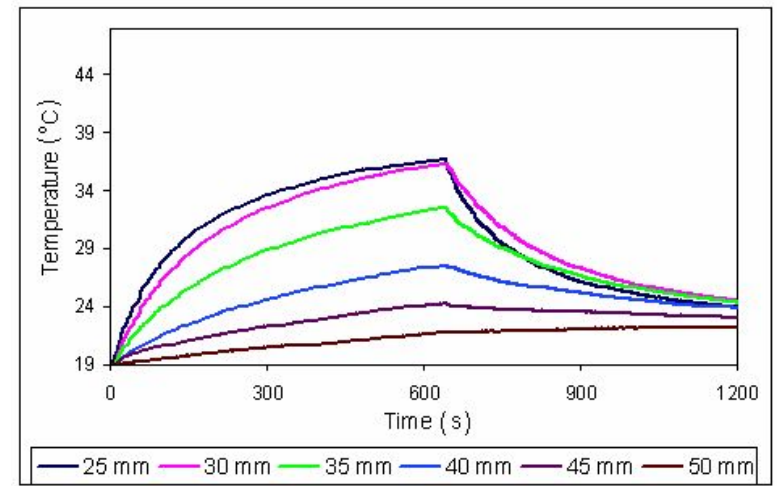

Fig. 4. Temperature variation in time measured in soft tissue by thermocouples along the acoustic axis at various distances from transducer, power applied $4 \mathrm{~W}$.

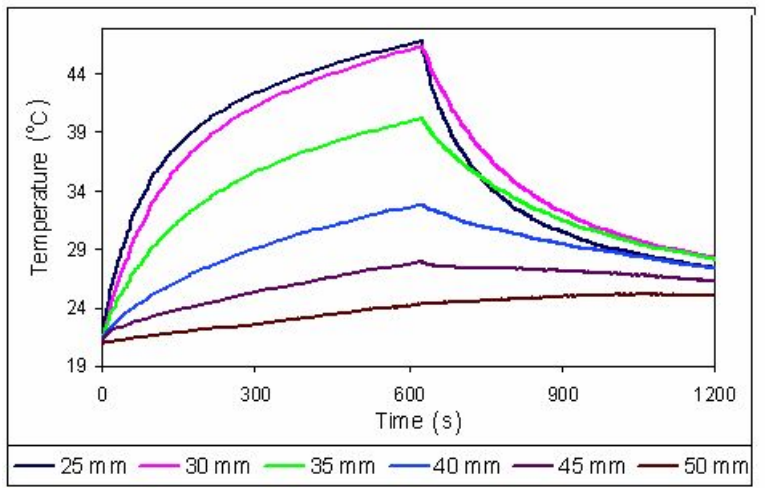

Fig. 5. Same as Fig. 4 but for power applied $6 \mathrm{~W}$.

Figures 4, 5, and 6 below illustrate the temperature changes at different distances from the transducer inside the sample and different power of heating. All calculations presented in this paper were done using MATLAB (The Mathworks Inc., Natick, Massachusetts, USA), version R2014a. 


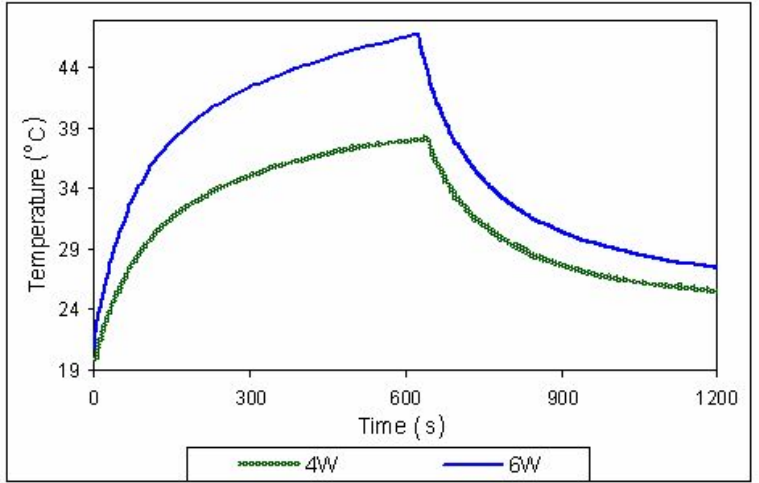

Fig. 6. Same as Figs. 4 and 5 but only at the focal point (the location with the highest heating effect).

\section{Methods of statistical analysis}

Three different probability distribution functions (PDFs), including one single-parameter (Rayleigh) and two two-parameter (Nakagami and $K$-distribution), have been used in statistical analysis. Sensitivity of $K$-distribution parameters is demonstrated in Fig. 7 .

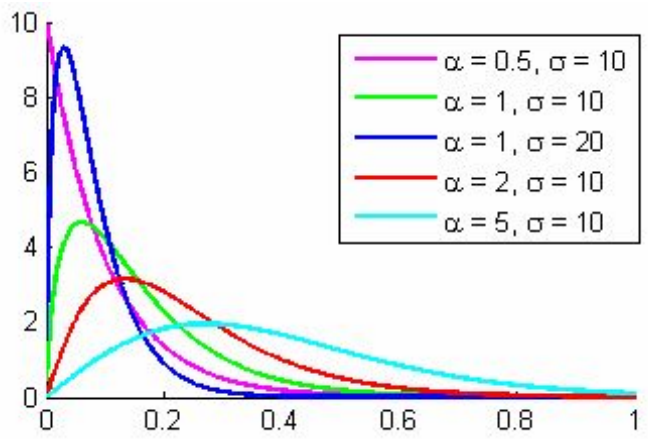

Fig. 7. The probability density function of the $K$ distribution, cf. Eq. (3.3).

The parameter of the Rayleigh distribution characterizes the intensity of the uniform isotropic identical scatterers distribution, called the diffuse scattering case. In the "point scattering model", this parameter is proportional to reflectivity of scatterers and is identified with their cross-sections [18]. Two-parameter PDFs (Nakagami and $K$-distribution) have the capacity to take into account the divergence from the Rayleigh distribution, i.e. from diffusive character of the scattering process [19].

The Rayleigh distribution is defined by

$$
P_{\mathrm{Ra}}(A)=\frac{A}{\sigma^{2}} \exp \left(-\frac{A^{2}}{2 \sigma^{2}}\right),
$$

whereas the Nakagami distribution is defined by

$$
P_{\mathrm{N}}(A)=\frac{2 m^{m}}{\Gamma(m) \Omega^{m}} A^{2 m-1} \exp \left(-m A^{2} / \Omega\right),
$$

where $m>0$ is the shape parameter, $\sigma>0, \Omega>0$ are scale parameters, and $\Gamma$ is the Euler gamma function.
The $K$-distribution is defined by

$$
P_{K}(A)=\frac{4 A^{\alpha}}{\left(2 \sigma^{2}\right)^{(\alpha+1) / 2} \Gamma(\alpha)} K_{\alpha-1}\left(\sqrt{\frac{2}{\sigma^{2}}} A\right),
$$

where $\alpha>0, \sigma>0$ are the shape parameter and the scale parameter, respectively, and $K_{p}$ denotes the modified Bessel function of the second kind of order $p$.

$\mathrm{RF}$ signals registered during the two experiments have been filtered to frequency band of $6-10 \mathrm{MHz}$. Next, for every fixed frame, analytic signals have been calculated and values of their envelopes have been normalized with respect to the maximum value.
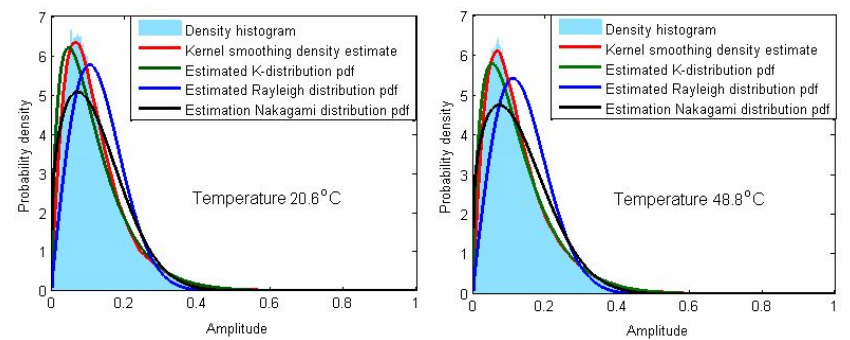

Fig. 8. The histogram determined for empirical data registered in the PVA-c phantom experiment for the initial (left part) and maximum (right part) temperature together with different probability density functions fitted to it.
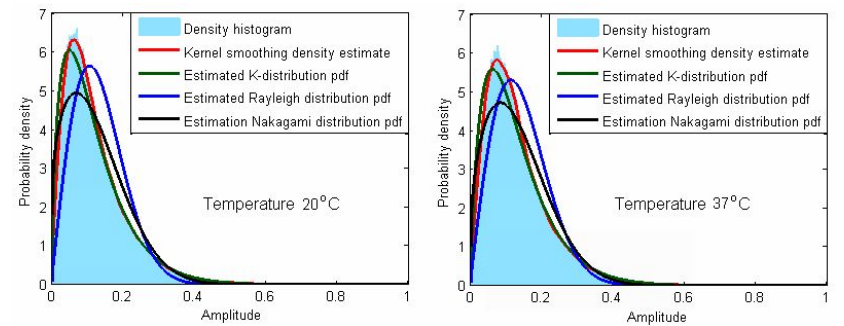

Fig. 9. The histogram determined for empirical data registered in the soft tissue sample experiment with heating power of $4 \mathrm{~W}$ for the initial (left part) and maximum (right part) temperature and probability density functions fitted to it.
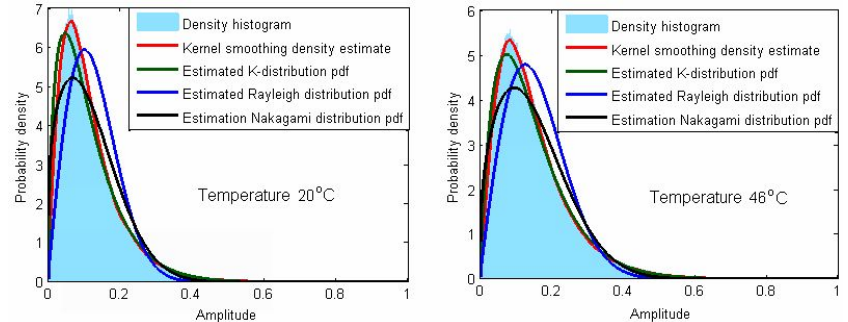

Fig. 10. Same as Fig. 9 but for heating power of $6 \mathrm{~W}$.

The observed data have been fitted, to the three PDFs using two different approaches. For the Rayleigh 
distribution and the Nakagami distribution, the parameters have been generated automatically in MATLAB software. For the $K$-distribution, the shape and scale parameters have been calculated by the methods of moments (cf. [1], Part 10). Histograms of envelopes fitted to different PDFs for all experiments at two points of the heating process, namely in the beginning and at the maximal temperature level, are shown in Figs. 8-10.

The goodness of fitting histograms to PDFs has been measured with the use of mean square error (MSE) calculations and the results are listed in Tables I-III.

TABLE I

The mean square error (MSE) determined by comparing the different distributions assigned to empirical data at different times during the experiment with heating and cooling of the PVA-c.

\begin{tabular}{c|c|c|c}
\hline \hline $\begin{array}{c}\text { Time } \\
{[\mathrm{min}]}\end{array}$ & $\begin{array}{c}\text { Rayleigh } \\
\text { distribution }\end{array}$ & $\begin{array}{c}\text { Nakagami } \\
\text { distribution }\end{array}$ & $K$-distribution \\
\hline 1 & 0.4027 & 0.1714 & 0.1207 \\
20 & 0.4887 & 0.1791 & 0.1028 \\
40 & 0.5188 & 0.2165 & 0.0991 \\
60 & 0.5150 & 0.1914 & 0.0670 \\
70 & 0.4622 & 0.1785 & 0.0663 \\
90 & 0.4223 & 0.1625 & 0.0597 \\
110 & 0.4366 & 0.1657 & 0.0647 \\
120 & 0.4483 & 0.1668 & 0.0647 \\
160 & 0.4438 & 0.1899 & 0.1049 \\
180 & 0.4433 & 0.1859 & 0.0956
\end{tabular}

TABLE II

Same as Table I but for the soft tissue sample and heating power of $4 \mathrm{~W}$.

\begin{tabular}{c|c|c|c}
\hline \hline $\begin{array}{c}\text { Time } \\
{[\mathrm{min}]}\end{array}$ & $\begin{array}{c}\text { Rayleigh } \\
\text { distribution }\end{array}$ & $\begin{array}{c}\text { Nakagami } \\
\text { distribution }\end{array}$ & $K$-distribution \\
\hline 1 & 0.4901 & 0.1734 & 0.056 \\
5 & 0.3526 & 0.1462 & 0.0414 \\
10 & 0.3030 & 0.1338 & 0.0512 \\
15 & 0.3890 & 0.1631 & 0.0498 \\
20 & 0.3929 & 0.1631 & 0.0528
\end{tabular}

TABLE III

Same as Table II but for heating power of $6 \mathrm{~W}$.

\begin{tabular}{c|c|c|c}
\hline \hline $\begin{array}{c}\text { Time } \\
{[\mathrm{min}]}\end{array}$ & $\begin{array}{c}\text { Rayleigh } \\
\text { distribution }\end{array}$ & $\begin{array}{c}\text { Nakagami } \\
\text { distribution }\end{array}$ & $K$-distribution \\
\hline 1 & 0.4656 & 0.1868 & 0.0893 \\
5 & 0.2763 & 0.1159 & 0.0399 \\
10 & 0.2675 & 0.1177 & 0.0279 \\
15 & 0.2962 & 0.1228 & 0.0324 \\
20 & 0.3397 & 0.1396 & 0.0346
\end{tabular}

The four parameters, which characterize the shape of considered distributions for both experiments as functions of time, are depicted in Figs. 11-14.
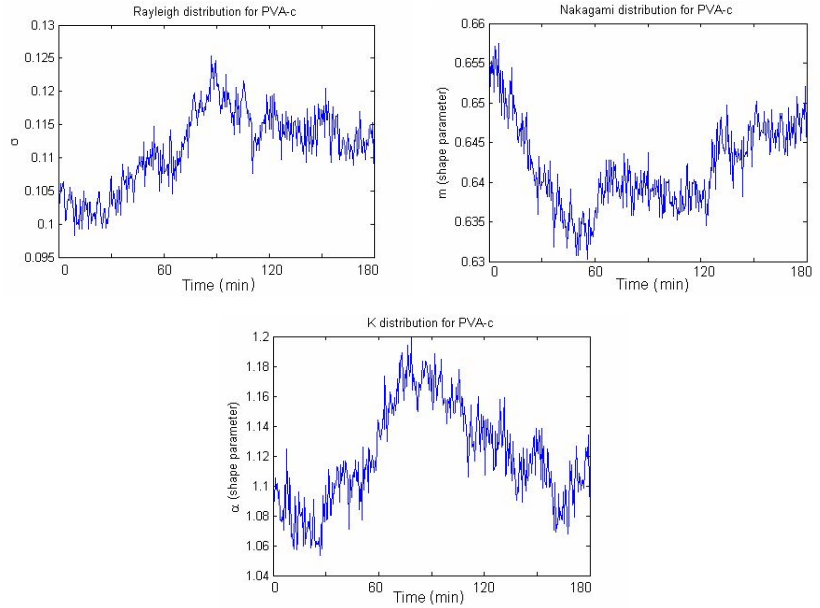

Fig. 11. The shape parameters of the Rayleigh distribution, the Nakagami distribution and the $K$ distribution as functions of time from data obtained for PVA-c sample experiment.

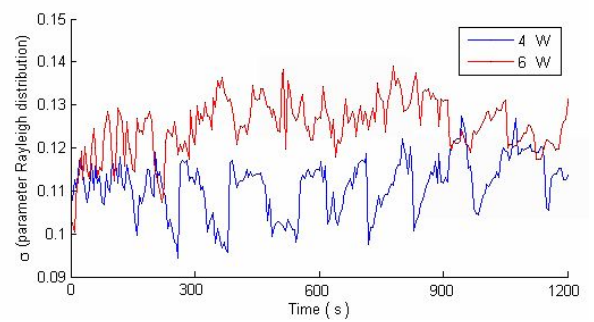

Fig. 12. The scale parameter $\sigma$ of the Rayleigh distribution as a function of time from data obtained for soft tissue sample and heating power of $4 \mathrm{~W}$ and $6 \mathrm{~W}$.

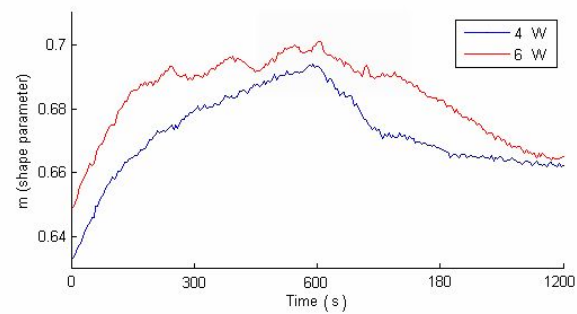

Fig. 13. The shape parameter of the Nakagami distribution as a function of time from data obtained in the soft tissue sample experiment for heating power of $4 \mathrm{~W}$ and $6 \mathrm{~W}$.

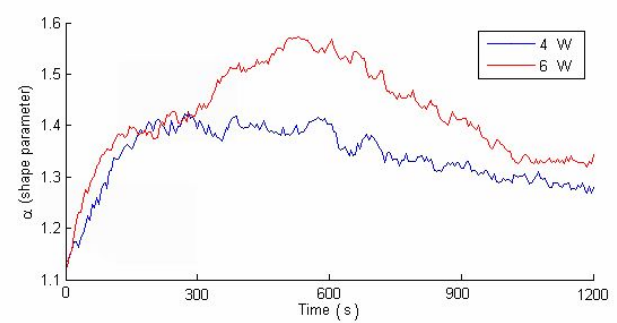

Fig. 14. The shape parameter of $K$-distribution as a function of time from data obtained in the soft tissue sample experiment for heating of power of $4 \mathrm{~W}$ and $6 \mathrm{~W}$. 


\section{Results}

To find a PDF parameter most suitable for evaluating the temperature changes, two criteria have been used: the histogram matching and the strongest sensitivity to temperature changes in different thermal processes and different materials samples. The $K$-distribution has not only the smallest MSE but also its shape parameter is the most accurate measure of the temperature increase/decrease. The MSE between histograms and $K$-distributions with parameters depending on time is one order of magnitude lower than in the two other cases. Moreover, the shape parameter of the $K$-distribution, unlike the Nakagami distribution shape parameter, can be attributed a physical interpretation as the so called "number of effective scatterers" in a resolution cell [19].

The linear regression has been used to obtain a smooth approximation. The rate of variation of the shape parameter is very close, to the rate of the measured temperature changes.
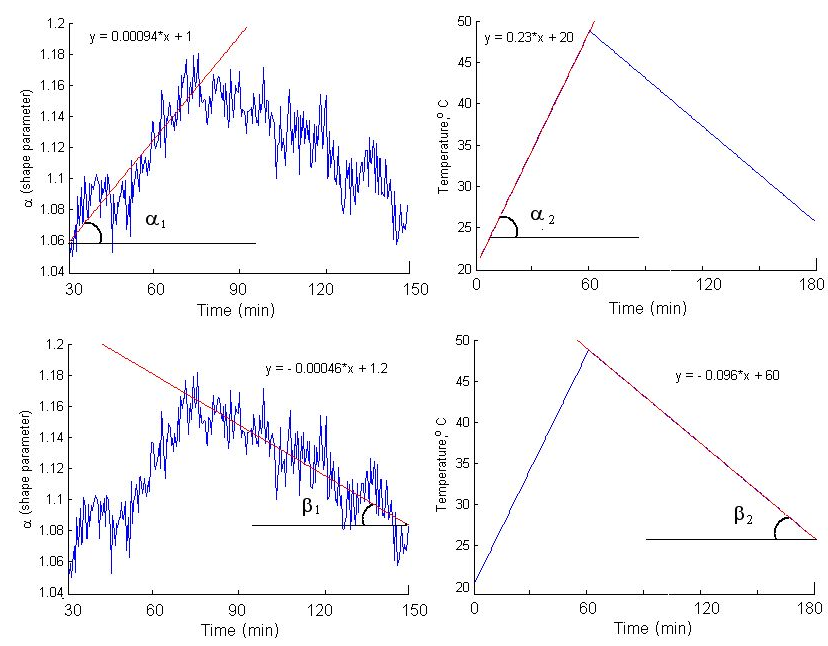

Fig. 15. Linear approximations of temperature changes in the shape parameter time dependence for PVA-c experiment (left parts) and the linear temperature function measured with thermometer (right parts).

Furthermore, the ratio of the rate of variation of the shape parameter in the heating regime to the rate of variation in the cooling regime is nearly equal to the same ratio of the measured temperature variation rates, cf. Fig. 15. The data obtained for PVA-c phantom corresponding to both the heating and the cooling process were processed by means of the linear regression method and the obtained inclination angles compared to those calculated from direct measurements, with the result represented by the following ratios:

$$
\frac{\tan \alpha_{1}}{\tan \beta_{1}}=\frac{0.00094}{0.00046} \approx 2, \quad \frac{\tan \alpha_{2}}{\tan \beta_{2}}=\frac{0.23}{0.096} \approx 2 .
$$

The links between the temperature changes during heating/cooling processes and the shape parameter changes are visualized in Fig. 16. The temperature colored scale has been calculated in agreement with temperature measured experimentally over time.
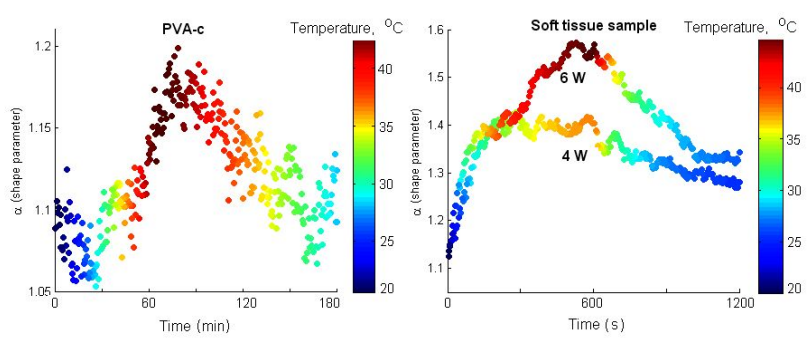

Fig. 16. The time/temperature relationship as a function of the $K$-distribution shape parameter for the PVA-c phantom (left part) and the soft tissue sample (right part).

\section{Conclusions}

It follows from the analysis presented above that to measure temperature changes in PVA-c sample, the shape parameter of the $K$-distribution has features of an excellent "thermometer". It measures not only the temperature level as a usual thermometer but also preserves the information about the shape of the time/temperature curve measured experimentally. It has been shown that the ratio of increase and decrease temperature rates measured by the shape parameter changes are consistent with experimental measurements.

The thermal process for PVA-c sample proceeded slowly (one hour of heating, two hours of cooling) compared to the process of heating the tissue sample in vitro (10 minutes of heating and 10 minutes of cooling). In such a heating process, the PVA-c sample was heated homogeneously by immersing in water whereas the soft tissue sample in vitro was heated locally, inside its volume, and the temperature distribution was strongly inhomogeneous. The two types of temperature/times curves derived for two values of heating power, $4 \mathrm{~W}$ and $6 \mathrm{~W}$ (cf. Fig. 6), are strongly nonlinear. It could be expected that variations of the shape parameter changes in the heating/cooling processes should be much larger. Surprisingly, the obtained results confirmed that the shape parameter of the $K$-distribution is acting as a "thermometer" or even better! In the case of PVA-c sample, we have demonstrated that this parameter can reflect not only temperature level changes but also the ratio of rates of the heating/cooling process. The perfect fit between the shape of nonlinear curves of Fig. 6 and the temperature dependence of the shape parameter of $K$ distribution in Fig. 16 could not occur accidentally. Tissue samples in vitro used in the experiment have properties similar to those of living soft tissues, and therefore the shape parameter can be used as a temperature marker of small volume of soft tissues in vivo. Although the results are very promising, they should be confirmed in the larger number of experiments carried out in repeatable conditions. 
The explanation of the physical phenomena that underlie our results is essential for the applicability of presented findings in a form of new non-invasive method of measuring the temperature inside the soft tissues in vivo.

\section{Acknowledgments}

This work was partially supported by the National Science Centre (grant no. 2011/03/B/ST7/03347).

\section{References}

[1] Quantitative Ultrasound in Soft Tissues, Eds. J. Mamou, M.L. Oelze, Springer, New York 2013.

[2] G. Ghoshal, A.C. Luchies, J.P. Blue, M.L. Oelze, J. Acoust. Soc. Am. 130, 4 (2011).

[3] R. Seip, E.S. Ebbini, IEEE T. Bio-Med. Eng. 42, 8 (1995).

[4] B. Gambin, T. Kujawska, E. Kruglenko, A. Mizera, A. Nowicki, Arch. Acoust. 34, 4 (2009).

[5] A. Mizera, B. Gambin, Commun. Nonlinear Sci. Numer. Simul. 16, 5 (2011).

[6] A. Mizera, B. Gambin, J. Theor. Biol. 265, 3 (2010).

[7] M. Vázquez, A. Ramos, L. Leija, A. Vera, Jpn. J. Appl. Phys 45, 1 (2009).
[8] S. Wang, V. Zderic, V. Frenkel, Future Oncol. 6, 9 (2010).

[9] C.H. Seo, Y. Shi, S.-W. Huang, K. Kim, M. O'Donnell, Interface Focus 1 (2011).

[10] B. Gambin, E. Kruglenko, Hydroacoustics 14, 99 (2011).

[11] B. Gambin, E. Kruglenko, T. Kujawska, M. Michajłow, Acta Phys. Pol. A 119, 950 (2011)

[12] E. Kruglenko, B. Gambin, L. Cieślik, Hydroacoustics 16, 121 (2013)

[13] B. Gambin, O. Doubrovina, Hydroacoustics 16, 59 (2013).

[14] E. Kruglenko, B. Gambin, Hydroacoustics, 17, 115 (2014).

[15] O. Doubrovina, B. Gambin, E. Kruglenko, Hydroacoustics 17, 37 (2014).

[16] Po-Hsiang Tsui, Yu-Chen Shu, Wen-Shiang Chen, Hao-Li Liu, Ing-Tsung Hsiao, Yu-Ting Chien, Med Phys. 39, 5 (2012).

[17] A. Nowicki, B. Gambin, Arch. Acoust. 39, 4 (2014).

[18] M. Pereyra, H. Batatia, IEEE T. Ultrason. Ferr. Freq Control 59, 1 (2012).

[19] F. Destrempes, G. Clouthier, Ultrasound Med. Biol. 36, 7 (2010). 